\title{
Reengineering valve patients' postdischarge management for adapting to bundled payment models
}

\author{
Michael S. Koeckert, MD, Patricia A. Ursomanno, PhD, NP, Mathew R. Williams, MD, \\ Michael Querijero, PA, Elias A. Zias, MD, Didier F. Loulmet, MD, Kevin Kirchen, MBA, \\ Eugene A. Grossi, MD, and Aubrey C. Galloway, MD
}

\begin{abstract}
Background: Bundled Payments for Care Improvement (BPCI) initiatives were developed by Medicare in an effort to reduce expenditures while preserving quality of care. Payment model 2 reimburses based on a target price for 90-day episode of care postprocedure. The challenge for valve patients is the historically high $(>35 \%$ ) 90-day readmission rate. We analyzed our institutional cardiac surgical service line adaptation to this initiative.
\end{abstract}

Methods: On May 1, 2015, we instituted a readmission reduction initiative (RRI) that included presurgical risk stratification, comprehensive predischarge planning, and standardized postdischarge management led by cardiac nurse practitioners (CNPs) who attempt to guide any postdischarge encounters (PDEs). A prospective database also was developed, accruing data on all cardiac surgery patients discharged after RRI initiation. We analyzed detailed PDEs for all valve patients with complete 30-day follow-up through November 2015.

Results: Patients included 219 surgical patients and 126 transcatheter patients. Sixty-four patients had 79 PDEs. Of these 79 PDEs, $46(58.2 \%)$ were guided by CNPs. PDEs were due to fluid overload/effusion (21, 27\%), arrhythmia (17, $22 \%$ ), bleeding/thromboembolic events $(13,16 \%)$, and falls/somatic complaints $(12,15 \%)$. Thirty-day readmission rate was $10.1 \%$ (35/345). Patients with transcatheter aortic valve replacement had a higher rate of readmission than surgical patients $(15.0 \%$ vs $6.9 \%)$, but were older with more comorbidities. The median readmission length of stay was 2.0 days (interquartile range 1.0-5.0 days). Compared with 2014, the 30-day readmission rate for BPCI decreased from $18 \%(44 / 248)$ to $11 \%(20 / 175), P=.05$.

Conclusions: Our reengineering of pre/postdischarge management of BPCI valve patients under tight CNP control has significantly reduced costly 30-day readmissions in this high-risk population. (J Thorac Cardiovasc Surg 2017;154:190-8)

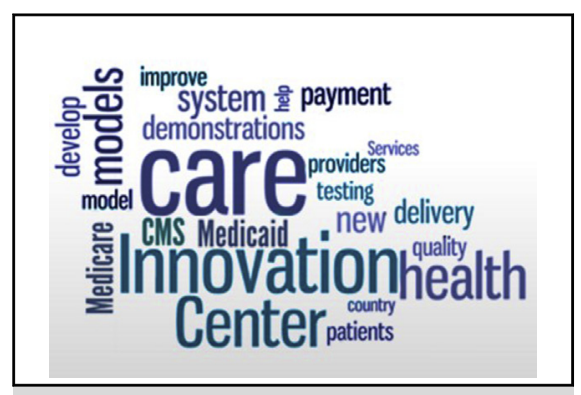

Logo for the CMS Innovation Center created by the Affordable Care Act.

\section{Central Message}

Surgeons must take ownership of patient management in the postdischarge period to survive in the era of financial risk bearing.

\section{Perspective}

The Affordable Care Act created an innovation center to explore solutions for health care cost reduction. What has been perceived as a postprocedural failure (high readmission rates after valves) has become a target of this reform. This has placed pressure on the cardiac surgeon to optimize postdischarge management with an institutional approach to avoid unnecessary and costly hospital readmissions.

See Editorial Commentary page 199.
The Centers for Medicare and Medicaid Services (CMS) has historically based reimbursement on a fee-for-service mechanism. However, this approach has been criticized as a major driver of health care costs as it contains

From the Department of Cardiothoracic Surgery, NYU-Langone Medical Center, New York, NY.

Read at the 42nd Annual Meeting of Western Thoracic Surgical Association, Waikoloa, Hawaii, June 22-25, 2016.

Received for publication June 23, 2016; revisions received Oct 11, 2016; accepted for publication Oct 26, 2016; available ahead of print April 12, 2017.

Address for reprints: Eugene A. Grossi, MD, Department of Cardiothoracic Surgery, NYU-Langone Medical Center, 530 First Ave, Suite 9V, New York, NY 10016 (E-mail: Eugene.grossi@nyumc.org).

$0022-5223 / \$ 36.00$

Copyright $\odot 2017$ by The American Association for Thoracic Surgery

http://dx.doi.org/10.1016/j.jtcvs.2016.10.109 unintended incentives for increasing the volume of care while not necessarily improving quality. ${ }^{1}$ In an effort to respond to these concerns, the Center for Medicare and Medicaid Innovation (CMMI) was created in 2010 by the Affordable Care Act (ACA) and instituted the Bundled Payments for Care Improvement (BPCI) initiative.

Scanning this QR code will take you to a supplemental video for the article. 


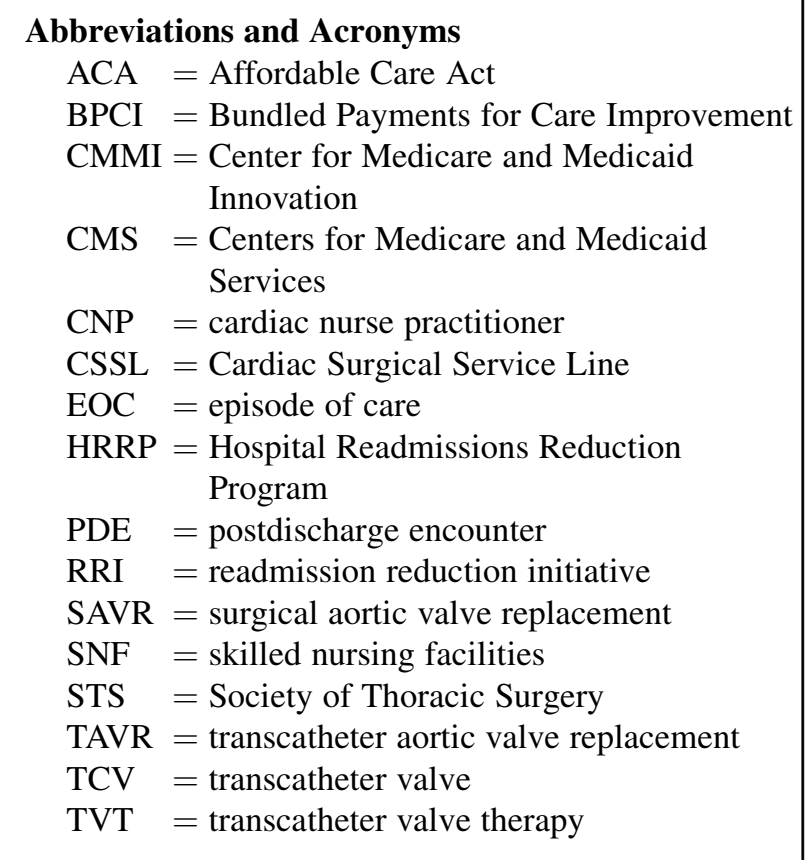

The BPCI program was developed to test payment and service delivery models with the potential to reduce expenditures during clinical episodes while preserving or enhancing the quality of care. ${ }^{2,3}$ Four different models are currently being tested that link payments for the multiple services rendered during a clinical care episode. An episode of care (EOC) is designated based on disease process or procedure as defined by disease-related group codes. BPCI model 2 EOCs include acute care and physician fees for the index procedure as well as related post-acute care, including readmissions and all related services through 90 days postdischarge. Model 2 includes a retrospective bundled payment arrangement in which actual expenditures are reconciled against a baseline target price for the EOC. The target price is set by CMS, individualized for an institution based on 3-year historic claims data, EOC category, episode length, and a $2 \%$ discount. For a "bundle" patient, the actual 90-day expenditures from the CMS master claims file are tabulated and reconciled. If total expenditures are below the target amount, the difference is paid to the institution; if above, recoupment is accordingly collected by CMS. Readmissions are therefore a costly penalty to the institution. Proponents of this model believe it will incentivize the more judicial use of health care resources and lead to less fragmentation of care. ${ }^{1,4,5}$

One of the challenges in caring for Medicare valve patients is the historically high ( $>40 \%$ ) 90-day readmission rates present in this elderly, high-risk population. ${ }^{6}$ It has been shown that between $50 \%$ and $80 \%$ of cardiac surgery readmissions occur in the first 30 days postdischarge. ${ }^{7}$ From a fiscal perspective, Medicare payments for unplanned rehospitalizations in 2004 accounted for approximately $\$ 17$ billion of the $\$ 100$ billion that Medicare awarded that year. ${ }^{8}$ Previously, extended postdischarge management and concomitant fiscal burden of late complications was typically beyond the surgeon's purview. Now that this expenditure has become a target for CMS penalty, this perspective has changed. We analyzed our institution's cardiac surgical service line (CSSL) adaptation to this BPCI model.

\section{METHODS \\ BPCI Details}

Our institution decided to participate for valve surgery in BPCI model 2 with a 90-day EOC. The phase 1 preparation period started in January 2013, with the financial risk-bearing phase starting in October 2013. Historically, before and during the preparation period, our discharge strategy had high reliance (approximately 70\%) on inpatient rehabilitation and skilled nursing facilities (SNFs), which was driven by length-of-stay concerns. Our initial approach to reduce postdischarge inpatient expenditures was to move away from facility-based post-acute care.

By the end of 2013, use of inpatient rehabilitation and SNFs was down to $21 \%$, resulting in beneficiary savings of more than $\$ 7000$ per patient. However, 30-day readmission remained at approximately $20 \%$.

\section{Implementation of Readmission Reduction Initiative}

In 2015, our cardiac service line team completed development of a comprehensive discharge planning and management approach. It begins with presurgical risk stratification based on a variety of heart failure, frailty, and cognitive measures (eg, Society of Thoracic Surgery [STS] risk score, Kansas City Cardiomyopathy Questionnaire, walk tests). Predischarge planning is then begun early in the postoperative course with patient and family education (emphasizing the importance of medication compliance), as well as coordination with home-care services or acute and subacute facilities. Standardized postdischarge management led by cardiac nurse practitioners (CNPs) begins with a phone call within 48 hours of discharge. CNPs then maintain weekly contact by phone and use telemedicine (Cardiocom, Chanhassen, Minn) to monitor the patient's clinical status and attempt to guide any postdischarge encounters (PDEs) throughout the first 30 days after discharge. Postdischarge office visits also have been regimented, with patients being seen on postdischarge days 7 and 21 and scheduled to see their cardiologist on day 14 . We also implemented a number of postdischarge triage and management protocols for patients presenting to our emergency department, which facilitates immediate evaluation by our team and standardizes the approach to common presentations, such as postoperative pain, atrial fibrillation, and volume overload. Whenever possible, less than 48-hour hospital observation was used instead of readmission. Additionally, a prospective database was created in May 2015 to track all adult cardiac surgery patients for 30 days postdischarge.

\section{Outcomes}

A retrospective analysis of our prospectively collected adult cardiac surgery discharge database was conducted. Additional data were collected from CMS claims as well as STS and transcatheter valve therapy (TVT) data. Local institutional review board approval for retrospective deidentified data analysis was obtained, and the requirement for written informed consent was waived.

PDE included any unplanned hospital encounter other than office visit, regardless of hospital setting; planned staged procedures were excluded. A patient was considered an inpatient readmission if the patient stayed more than 48 hours in-house, as per CMS definition. For less than 48 hours, the patient was classified as observational or outpatient status. 


\section{Statistics}

Statistical analyses were performed using SPSS 22 (IBM Corp, Armonk, NY). Encounter rate and readmission rate were calculated per index discharge. Continuous variables are reported as mean \pm standard deviation and skewed data as median and interquartile range (IQR), unless otherwise stated. Categorical variables are reported as a rate of occurrence. Groups were compared by $\chi^{2}$ methods, Fisher exact tests, and analysis of variance. A $P$ value less than .05 was considered statistically significant. Where appropriate, post hoc analysis was performed for contingency tables, and $P$ values between groups were calculated by using the partitioning method and a Bonferroni correction was applied.

\section{RESULTS \\ Patients}

From May through November 2015, 345 valve patients were discharged; 219 open surgical patients (Open) and 126 transcatheter valve patients (TCV). The TCV patients were predominantly transcatheter aortic valve replacements (TAVRs) but also included 3 transcatheter mitral valve replacements. Of the Open valves, there were 89 isolated aortic valve replacements, 72 mitral valve repair or replacements, 25 multivalve cases, 17 combined valve/coronary bypasses, and 16 others.

The average age was $71 \pm 14$ years and $63.8 \%$ were men (220/345). STS risk score ranged from $0.2 \%$ to $28.0 \%$ with a median of $4.2 \%$ (IQR $1.0 \%-6.3 \%$ ) and $51.9 \%$ of patients were in New York Heart Association heart failure class III or IV. Demographic data are summarized in Table 1. TCV patients were older $(82.0 \pm 8.6$ years vs $65.0 \pm 12.9$ years,
$P<.001$ ) and had a higher STS risk score of $6.6 \%$ (IQR 5.2-8.8), $P<.001$. Additionally, TCVs had worse heart failure, twice the incidence of several major comorbidities, and were frailer (Table 1). The median length of stay postprocedure was 2 days (IQR 1-2 days) for TCV patients and 5 days (IQR 4-7 days) for Open patients $(P<.001)$. Discharge home with health services occurred in 80.3\% (377/345). There was a significant difference in discharge patterns with more Open patients $(9.6 \%)$ requiring inpatient rehabilitation than TCV patients (2.4\%) (Table 2).

\section{Postdischarge Encounters}

Sixty-four patients $(18.6 \%)$ had 1 or more PDE. A total of 79 PDEs occurred for a rate of PDE per index discharge of $22.9 \%$. Fourteen patients $(4.1 \%)$ had more than 1 PDE, with $13(3.8 \%)$ having 2 PDEs and 1 patient having 3 PDEs. The PDEs included 40 observational stays, 4 (nonoffice) outpatient visits, and 35 (44.2\%) inpatient readmissions. The total readmission rate was $10.1 \%(35 / 345) ; 2$ patients had 2 readmissions. TCV patients had a higher readmission rate of $15.9 \%(20 / 126)$ versus $6.8 \%(15 / 219)$ among Open patients, $P=.035$. Median length of stay for readmissions was 4 (IQR 3-7) days for transcatheter patients and 6 (IQR 3-6) days for open surgical patients. One readmitted patient died from a stroke after readmission to an outside hospital on postdischarge day 21 .

TABLE 1. Demographics

\begin{tabular}{|c|c|c|c|c|}
\hline Characteristic & $\begin{array}{c}\text { All valve patients, } \\
\mathbf{n}=\mathbf{3 4 5}, \mathbf{n}(\%)\end{array}$ & $\begin{array}{l}\text { Transcatheter, } \\
\mathbf{n}=126, \mathbf{n}(\%)\end{array}$ & $\begin{array}{l}\text { Open surgical, } \\
\mathbf{n}=219, \mathbf{n}(\%)\end{array}$ & $P$ \\
\hline Age, y & $71.3 \pm 14.2$ & $82.3 \pm 8.6$ & $65.0 \pm 12.9$ & $<.001$ \\
\hline Male gender & $220(63.8)$ & $81(64.3)$ & $139(63.5)$ & .913 \\
\hline White race & $298(86.4)$ & 117 (92.9) & $181(82.6)$ & .008 \\
\hline BMI, $\mathrm{kg} / \mathrm{m}^{2}$ & $27.5 \pm 6.2$ & $27.2 \pm 5.5$ & $27.8 \pm 6.6$ & .365 \\
\hline STS risk score, $\%$ & & & & $<.001$ \\
\hline Median (IQR) & $3.0(1.0-6.25)$ & $6.6(5.2-8.8)$ & $1.4(0.7-2.8)$ & \\
\hline Ejection fraction, $\%$ & $57.5 \pm 12.6$ & $55.8 \pm 14.3$ & $58.5 \pm 11.4$ & .080 \\
\hline NYHA III-IV & 179 (51.9) & $108(85.7)$ & $71(32.4)$ & $<.001$ \\
\hline Katz index & $5.7 \pm 0.9$ & $5.4 \pm 1.2$ & $5.9 \pm 0.7$ & $<.001$ \\
\hline Lives alone & $95(27.5)$ & $39(31.0)$ & $56(25.6)$ & .256 \\
\hline Two falls $<6$ mo & $10(2.9)$ & $8(6.3)$ & $2(0.9)$ & .006 \\
\hline Prior sternotomy & $66(19.1)$ & $41(32.5)$ & $25(11.4)$ & $<.001$ \\
\hline Atrial fibrillation & $90(26.1)$ & $36(28.6)$ & $54(24.7)$ & .446 \\
\hline Diabetes & $86(24.9)$ & $40(31.7)$ & $46(21.0)$ & .026 \\
\hline COPD & $61(17.7)$ & $30(23.8)$ & $31(14.2)$ & .024 \\
\hline PVD & $38(11.0)$ & $26(20.6)$ & $12(5.5)$ & $<.001$ \\
\hline CKD & $77(22.3)$ & $44(34.9)$ & $33(15.1)$ & $<.001$ \\
\hline CVA & $29(8.4)$ & $13(10.3)$ & $16(7.3)$ & .332 \\
\hline Coronary artery disease & $157(45.5)$ & $84(66.7)$ & $73(33.3)$ & $<.001$ \\
\hline BPCI participant & $175(50.7)$ & $84(66.7)$ & $91(41.6)$ & $<.001$ \\
\hline Index postoperative LOS, d, median (IQR) & $4(2-6)$ & $2(1-2)$ & $5(4-7)$ & $<.001$ \\
\hline 30-d mortality & $3(0.9)$ & $1(0.8)$ & $2(0.9)$ & 1.000 \\
\hline
\end{tabular}

BMI, Body-mass index; STS, Society of Thoracic Surgeons; IQR, interquartile range; NYHA, New York Heart Association; COPD, chronic obstructive pulmonary disease; $P V D$, peripheral vascular disease; $C K D$, chronic kidney disease; CVA, cerebral vascular accident; $B P C I$, Bundled Payments for Care Improvement; $L O S$, length of stay. 
TABLE 2. Discharge disposition

\begin{tabular}{|c|c|c|c|c|}
\hline Discharge disposition & $\begin{array}{l}\text { All valve patients, } \\
\mathbf{n}=\mathbf{3 4 5}, \mathbf{n}(\%)\end{array}$ & $\begin{array}{l}\text { Transcatheter, } \\
\mathbf{n}=\mathbf{1 2 6}, \mathbf{n}(\%)\end{array}$ & $\begin{array}{l}\text { Open surgical, } \\
\mathbf{n}=\mathbf{2 1 9}, \mathbf{n}(\%)\end{array}$ & $P^{*}$ \\
\hline & & & & .042 \\
\hline Home & $32(9.3)$ & $9(7.1)$ & $23(1.5)$ & .300 \\
\hline Home with services & $277(8.3)$ & $110(87.3)$ & $167(76.3)$ & .013 \\
\hline Inpatient rehabilitation & $24(7.0)$ & $3(2.4)$ & $21(9.6)$ & .011 \\
\hline Skilled nursing facility & $12(3.5)$ & $4(3.2)$ & $8(3.7)$ & .815 \\
\hline
\end{tabular}

* $P$ value for contingency table listed first. Bonferroni correction applied with significant $P<.013$.

The median time to PDE was 11 days (IQR 6-19) and $35.4 \%$ (28/79) occurred during the first week after discharge. Of these first-week encounters, $35.7 \%$ resulted in readmission (10/28). Subsequently, PDEs and readmissions were distributed equally across postdischarge weeks 2 to 4 (Tables 3 and 4). The time to reencounter and readmission were not different between groups.

Indication for PDE is summarized in Figure 1. The most common reason for PDE was fluid overload or effusion (21/ $79,26.6 \%$ ) followed by arrhythmia $(17 / 79,21.5 \%)$, somatic pain $(9 / 79,11.4 \%)$, and thromboembolic events (8/ $79,10.1 \%)$. Other indications for reencounter included gastrointestinal disease $(6 / 79,7.6 \%)$, infection $(5 / 79$, $6.3 \%)$, bleeding $(5 / 79,6.3 \%)$, falls $(3 / 79,3.8 \%)$, and other indications $(5 / 79,6.3 \%)$. Fluid overload encounters resulted in readmission $57.1 \%$ of the time (12/21), whereas PDE for arrhythmia resulted in readmission $29.4 \%$ of the time (5/17). PDEs were more likely to be associated with arrhythmia in Open patients, whereas PDEs were more associated with volume overload in TCV patients (Figure 2).

CNPs guided 58.2\% (46/79) of PDEs. If the encounter was guided, the patient was more likely to present to our institution rather than an outside hospital, $74 \%$ versus $33 \%$ of nonguided encounters $(P<.001)$. Compared with 2014, the 30-day readmission rate for BPCI decreased from $18 \%(44 / 248)$ to $11 \%(20 / 175), P=.05$.

\section{DISCUSSION}

The Hospital Readmissions Reduction Program (HRRP) was created with CMMI as part of the ACA in $2010 .{ }^{10} \mathrm{~A}$ primary motivator for this reform was the article by Jencks and colleagues $^{8}$ in the New England Journal of Medicine the year prior that estimated $\$ 17.4$ billion was spent by Medicare on 30-day readmissions; this made 30-day readmission reduction an opportune reform target. To that end, HRRP has begun to implement financial penalties for institutions having a higher-than-average 30-day readmission rate for selected clinical conditions. Although the purview of these financial penalties has not yet been extended to include readmission after cardiac surgery, this is widely expected to be forthcoming. Due to this concern, many institutions, including our own, have implemented readmission reduction strategies in patients after cardiac surgery.

Historically, cardiac surgeons have left much of the postdischarge management in the hands of cardiologists. As at many tertiary centers relying on a large referral base, many private physicians following our patients are not part of our network. This typically leaves little control over patient management choices and limits the ability to accurately track outcomes. Our early reduction of postdischarge SNF utilization avoided the scenario in which these facilities would just transfer back to a local emergency room when any complication occurred, thus preventing the opportunity for our outpatient management. Therefore, our readmission reduction initiative (RRI) program was created with the focus of maintaining ownership of our patients after discharge. By creating a regimented postdischarge process by using frequent phone calls and multiple office visits starting within the first week of discharge, we demonstrated success at early identification of many postoperative issues and treating them efficiently without readmission. With close follow-up and a $24 / 7$ oncall hotline, we also have had success in guiding patients in need of care escalation to the hospital, specifically to NYU. Directing patients to our institution is not only safer

TABLE 3. Time to postdischarge encounter

\begin{tabular}{lcrr}
\hline Time to postdischarge encounter & $\begin{array}{c}\text { All valve patients, } \\
\mathbf{n}=\mathbf{7 9 ,} \mathbf{n}(\%)\end{array}$ & $\begin{array}{c}\text { Transcatheter, } \\
\mathbf{n}=\mathbf{3 8}, \mathbf{n}(\%)\end{array}$ & $\begin{array}{c}\text { Open surgical, } \\
\mathbf{n}=\mathbf{4 1}, \mathbf{n}(\%)\end{array}$ \\
\hline Week 1 & $28(35.4)$ & $13(34.2)$ & $15(36.6)$ \\
Week 2 & $18(22.8)$ & $10(26.3)$ & $8(19.5)$ \\
Week 3 & $17(21.5)$ & $5(13.2)$ & $12(29.3)$ \\
Week 4 & $16(20.3)$ & $10(26.3)$ & $6(14.6)$ \\
Median (interquartile range), d & $11(6-19)$ & $10.5(6-23)$ & $13(6-17)$ \\
\hline
\end{tabular}


TABLE 4. Time to readmission

\begin{tabular}{|c|c|c|c|c|}
\hline Time to readmission & $\begin{array}{l}\text { All valve patients, } \\
\mathbf{n}=35, \mathbf{n}(\%)\end{array}$ & $\begin{array}{l}\text { Transcatheter, } \\
\mathbf{n}=\mathbf{2 0 ,} \mathbf{n}(\%)\end{array}$ & $\begin{array}{l}\text { Open surgical, } \\
\mathbf{n}=15, \mathbf{n}(\%)\end{array}$ & $P$ \\
\hline Week 1 & $10(35.7)$ & $6(30.0)$ & $4(26.7)$ & .389 \\
\hline Week 2 & $6(17.1)$ & $4(20.0)$ & $2(13.3)$ & \\
\hline Week 3 & $9(25.7)$ & $3(15.0)$ & $6(40.0)$ & \\
\hline Week 4 & $10(28.6)$ & $7(35.0)$ & $3(20.0)$ & \\
\hline Median (interquartile range), $\mathrm{d}$ & $15(7-23)$ & $14.5(6.5-25.5)$ & $16(7-20)$ & .826 \\
\hline
\end{tabular}

in terms of continuity of care, but is also likely to be much more cost-effective by obviating the need for redundant diagnostic tests and workup.

Overall, we found a 30-day PDE rate of $22.9 \%$ with a readmission rate of $10.1 \%$. The median time to PDE and readmission was 11 and 15 days, respectively, with approximately one-third of all PDEs occurring in the first week. One concern was that PDEs could be affected by shorter length-of-index admission, ${ }^{11}$ but this is not obviously true from our experience. Readmission rate for the TCV patients was higher than for Open cases, $15.9 \%$ and $6.8 \%$, respectively; however, the transcatheter patients represent an older and sicker population. Although the transcatheter patients were crudely differentiated in this initial analysis, in-depth study of this cohort is pending and may afford insight as to which patient-specific features are associated with readmission. We identified 2 major sources for PDE and readmission, fluid overload/heart failure and arrhythmic issues, that together account for almost half of all PDEs and readmissions, with arrhythmia more frequent among Open patients. Hopefully, as we accrue more data, we will gain insight on how to predict these complications.

Similar to some of our findings, a recent study by Hannan and colleagues ${ }^{12}$ analyzed 30-day readmission for TAVR/ SAVR by using New York's administrative acute-care database. With multiple patient exclusions, emergency room visit exclusion, and propensity matching, 30-day readmissions between SVAR and TAVR groups were similar at $18.8 \%$ and $19.3 \%$; the most common reasons for readmission

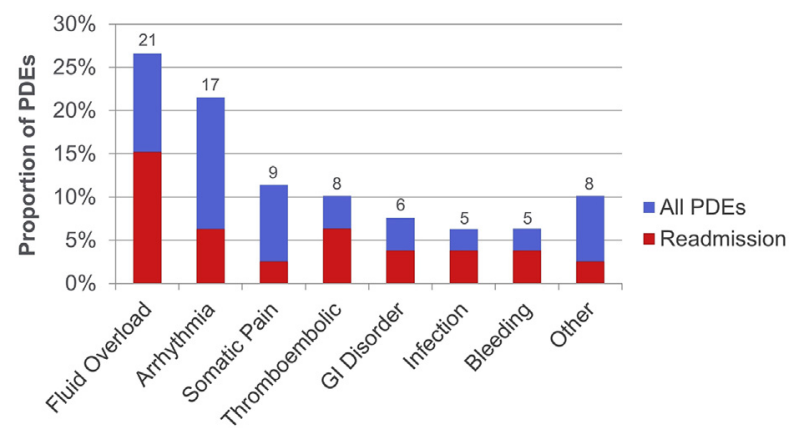

FIGURE 1. Postdischarge encounter and readmission rates. PDE, Postdischarge encounter; GI, gastrointestinal. were heart failure and arrhythmia. Iribarne and colleagues ${ }^{7}$ recently published readmission data from the Cardiothoracic Surgery Trials Network of more than 5000 open surgical patients followed for 65 days postdischarge. Readmission rate was $18.7 \%$ overall, with $80 \%$ occurring in the first 30 days. Rates for valve surgery and valve/coronary artery bypass grafting were $18.3 \%$ and $24.0 \%$. For this study, they did not distinguish between observation and inpatient status, excluded emergency room visits, and included patients with ventricular assist devices, who had the highest readmission rate $(>35 \%)$.

One of the major challenges of bundled payment models (particularly for such high-risk EOC as cardiac surgery) is the random variation in episode spending that can occur, although one method of correcting this is the use of risk stratification. However, Yount and colleagues ${ }^{13}$ recently showed that using the STS mortality risk score, the most validated surgical risk score available, did not adequately predict variation in costs. If bundled payment models are to succeed, particularly for cardiac valve surgery, more sophisticated tools must be developed to adjust risk and predict resource consumption.

\section{Limitations}

There were several limitations to this study. This is a single-center analysis of our initial process results with a relatively small sample size. Before the initiation of our RRI and associated database, the availability of readmission data is scant and we relied on CMS claims data for a control group. Although we had the benefit of our electronic

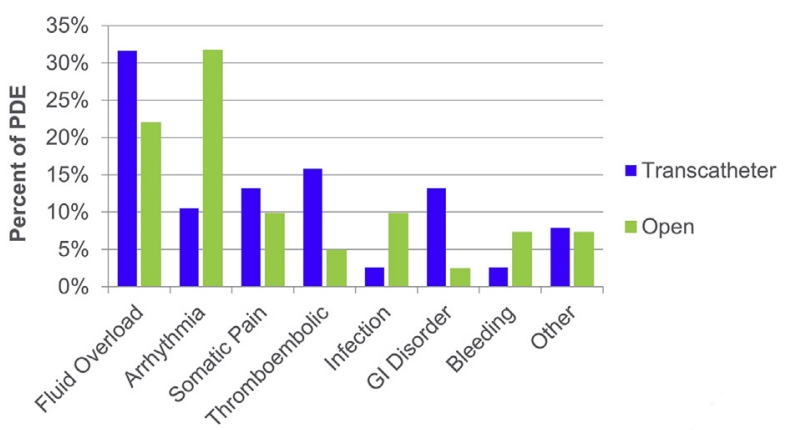

FIGURE 2. Distribution of PDE indication by procedure. PDE, Postdischarge encounter; GI, gastrointestinal. 


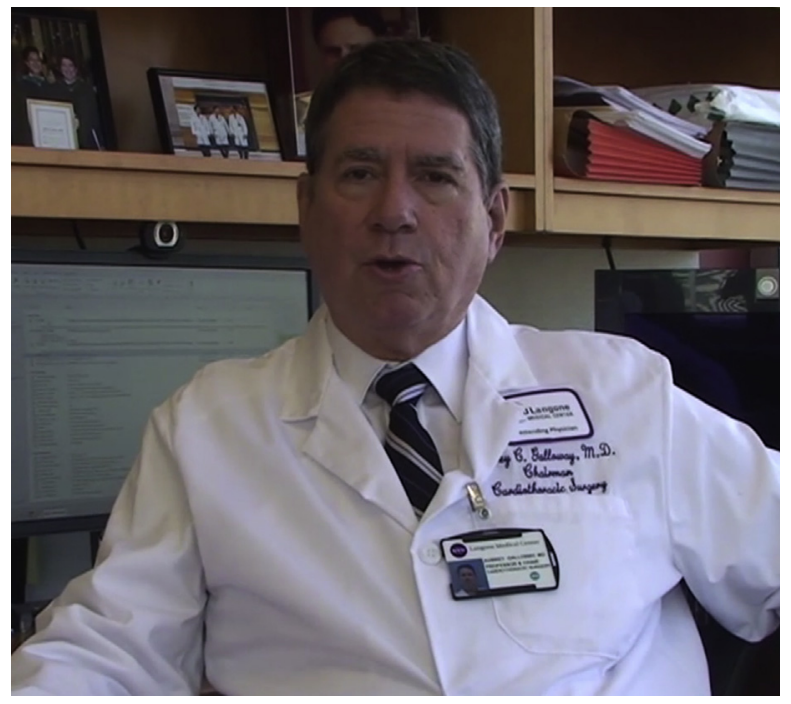

VIDEO 1. The senior author (A.C.G.) discussing the significance of the Bundle Initiative and impact on cardiothoracic practice. Video available at: http://www.jtcvsonline.org/article/S0022-5223(17)30403-8/addons.

medical record for patients seen at our institution, the accuracy of outside hospital claims data may be unreliable.

\section{CONCLUSIONS}

Our approach of reengineering postdischarge management of BPCI valve patients under tight cardiac surgical service line and CNP control has significantly reduced costly 30-day readmissions in this high-risk population. See Video 1 for commentary. We must take ownership of the postdischarge period to survive in the era of financial risk sharing and we cannot rely on administrative databases, but must create our own database and processes.

The full fiscal impact of this strategy on BPCI valve patients will be understood next year when the 90-day CMS master claims data are reconciled. Additionally, further data and analysis may allow focusing postdischarge management resources on those patients identified at high risk for 30/90-day readmissions.

\section{Conflict of Interest Statement}

Authors have nothing to disclose with regard to commercial support.

\section{References}

1. Schroeder SA, Frist W. National Commission on Physician Payment R. Phasing out fee-for-service payment. N Engl J Med. 2013;368:2029-32.

2. Centers for Medicare and Medicaid Services. Bundled payments for care improvement (BPCI) initiative. Available at: https://innovation.cms.gov/ initiatives/bundled-payments/. Accessed April 1, 2016.

3. American Hospital Association. Medicare's bundled payment initiatives: considerations for providers. Available at: http://www.aha.org/content/16/issbriefbundledpmt.pdf. Accessed April 1, 2016.

4. Hussey PS, Eibner C, Ridgely MS, McGlynn EA. Controlling U.S. health care spending - separating promising from unpromising approaches. $N$ Engl J Med. 2009;361:2109-11.
5. Mechanic RE, Altman SH. Payment reform options: episode payment is a good place to start. Health Aff (Millwood). 2009;28:w262-71.

6. Fry DE, Pine M, Nedza SM, Locke DG, Reband AM, Pine G. Inpatient and 90day postdischarge outcomes in cardiac surgery. Am J Account Care. 2016;3: $16-21$.

7. Iribarne A, Chang H, Alexander JH, Gillinov AM, Moquete E, Puskas JD, et al. Readmissions after cardiac surgery: experience of the National Institutes of Health/Canadian Institutes of Health research cardiothoracic surgical trials network. Ann Thorac Surg. 2014;98:1274-80.

8. Jencks SF, Williams MV, Coleman EA. Rehospitalizations among patients in the Medicare fee-for-service program. N Engl J Med. 2009;360:1418-28.

9. Jubelt LE, Goldfeld KS, Chung W, Blecker SB, Horwitz LI. Changes in discharge location and readmission rates under Medicare bundled payment. JAMA Intern Med. 2016;176:115-7.

10. Centers for Medicare and Medicaid Services. Readmissions reduction program Available at: http://www.cms.gov/Medicare/medicare-fee-for-service-payment/ acuteinpatientPPS/readmissions-reduction-program.html. Accessed April 1, 2016.

11. Zuckerman RB, Sheingold SH, Orav EJ, Ruhter J, Epstein AM. Readmissions, observation, and the Hospital Readmissions Reduction Program. N Engl J Med. 2016;374:1543-51.

12. Hannan EL, Samadashvili Z, Jordan D, Sundt TM, Stamato NJ, Lahey SJ, et al Thirty-day readmissions after transcatheter aortic valve implantation versus surgical aortic valve replacement in patients with severe aortic stenosis in New York State. Circ Cardiovasc Interv. 2015;8:e002744.

13. Yount KW, Isbell JM, Lichtendahl C, Dietch Z, Ailawadi G, Kron IL, et al Bundled payments in cardiac surgery: is risk adjustment sufficient to make it feasible? Ann Thorac Surg. 2015;100:1646-52.

Key Words: health economics, valve disease

\section{Discussion}

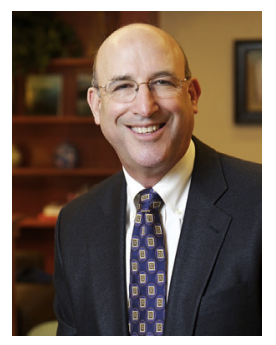

DISCUSSANT: Dr Robin Cohen

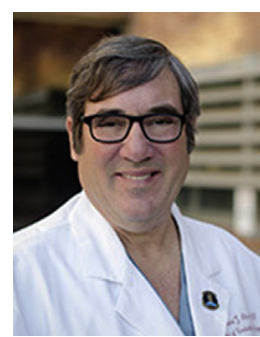

DISCUSSANT 2: Dr Richard Shemin

\section{MODERATOR: Dr James I. Fann}

\section{PRESENTER: Dr Michael Koeckert}

Dr Fann. The next presentation is entitled, "Reengineering of Medicare Valve Patients, Postdischarge, 30-Day Clinical Management; Successful Strategies for Adapting to Bundled Payment Models," to be presented by Michael Koeckert. Coauthors include Patricia Ursemile, Matthew Williams, and their colleagues at NYU.

Dr Koeckert. Good morning and Aloha. Thank you for the opportunity to present "Reengineering of Valve 
Patients, Postdischarge, 30-Day Clinical Management; Successful Strategies for Adapting to Bundled Payment Models." We have no relevant disclosures. Traditionally, Medicare is reimbursed based on a fee-for-service model; however, this model has been criticized as a major driver of health care costs, containing unintended incentives for increasing the volume of care while not necessarily improving quality. To respond, the Bundled Payments for Care Improvement initiative, or BPCI, was instituted by the Center for Medicare and Medicaid Innovation. Under BPCI, CMS is testing 4 payment models, which linked reimbursement for the multiple services rendered during an episode of care. We chose to participate in model 2, which is a retrospective payment model in which actual expenditures are reconciled against a target price for an episode of care.

Under model 2, for valve procedures, the episode includes the index, surgery, and hospitalization, physician fees, and readmissions at any hospital for up to 90 days after discharge. The target price, or bundled payment amount, is set by the Centers for Medicare and Medicaid Services (CMS) and individualized based on procedure and institution, based on historic 3-year claims data. When total claims expenditures are reconciled against this target price, CMS will either make a payment or take recoupment for the difference. Presented here is a timeline of our postdischarge management and participation in the BPCI program. Historically, a major concern for the hospital was optimizing length of stay. To that end, we relied heavily on the use of inpatient rehabilitation and skilled nursing facilities. Our initial approach to reduce postdischarge expenditures was to move away from this facility-based post-acute care. By the end of 2013, the use of inpatient rehabilitation and SNF was down from $70 \%$ to $21 \%$, resulting in beneficiary savings of more than $\$ 7000$ per patient. However, throughout this period, 30-day readmission rates remained high at approximately $20 \%$. Our participation in the BPCI program began with phase 1 , the preparation period, in January 2013. In October 2013, phase 2, or the risk-bearing period began, in which we assumed financial liability for the 90-day episode of care.

As a means of adapting to the bundled payment model, we initiated a readmission reduction program in May of 2015. We reengineered our approach to discharge planning and postdischarge management with the hope of reducing costly 30-day readmissions. This brings us to the purpose of this study, which is to analyze the impact of these preand postdischarge planning and management protocols on 30-day readmission rates for all valve patients. Our readmission reduction initiative or RRI, began in May of 2015. It includes presurgical risk stratification based on a variety of validated heart failure and frailty measures, comprehensive predischarge planning, with patient and family education, as well as coordination with home-care services or acute and subacute facilities, standardized postdischarge management led by cardiac nurse practitioners (NPs), emergency department rapid triage, and care protocols to enable quick assessment by our team and minimize unnecessary testing or admission, and, finally, a prospective outcomes database tracking all patients for 30 days after discharge.

This is a brief summary of our postdischarge management. Patients receive a phone call within 48 hours of discharge and are subsequently seen in the office on postdischarge days 7 and 21 . On day 14, they're seen by their private cardiologists and receive a second call from our cardiac NPs. Finally, they receive a graduation call on day 31 . Concurrently, we collect tele health data throughout the 30-day period, which includes daily heart rate, blood pressure, weight, and pain scores as assessed by the home health provider or patient. Any red flag values are reported to our NPs. For this analysis, we used our prospective RRI database as well as STS and TVT data. We included all adult valve patients discharged from May through November of 2015 . We analyze data by procedure type, transcatheter versus open, and perform subgroup analysis of BPCI participants. Our outcomes of interest were the rates of post discharge encounter and inpatient readmission. A postdischarge encounter, or PDE, is any unplanned hospital encounter. This can include outpatient emergency department visits, observational stays, which is defined by CMS as less than 2 midnights, and inpatient readmissions, which are the subject of CMS penalties.

During our study period, 345 valve patients were discharged, 126 transcatheter patients, who were predominantly TAVR, and 219 open surgical valves among which there were 89 AVRs, 72 micros, and other cases as listed. This busy slide shows our patient demographics. As expected, transcatheter patients were older, 82 versus 65 years old, and at higher STS risk scores. Additionally, they had worse heart failure, twice the incidents of several major comorbidities, and were frailer. Index procedure medium length of stay was 2 days for transcatheter patients and 6 days for open surgical patients. Graph here is discharge disposition. The great majority of patients were discharged home with health services. There was a small difference in discharge patterns, with more transcatheter patients going home with services, whereas more open valves required inpatient rehabilitation. Postdischarge encounter and readmission rates are shown here. Each yellow bar represents the rate of all PDEs. The hash pattern shows the proportion of true inpatient readmissions.

Overall, 64 patients had a total of 79 PDEs, of which 35 were inpatient readmissions, giving a rate of PDE per index discharge of $23 \%$ and a readmission rate of $10 \%$. Both PDE rate and readmission rate were higher in the transcatheter group but only the readmission rate was different significantly with a rate of $16 \%$ among transcatheter patients 
and $7 \%$ for open patients. Half of all PDEs lasted more than 1 day. Medium length of stay for readmission was 4 days for transcatheter patients and 6 days for open surgical patients. Approximately one-third of patients presented in the first week after discharge. The remaining PDEs were distributed equally throughout the rest of the month. The time to reencounter was not different between groups. Indications for PDE and readmission are shown here. Again, the yellow is the percentage of all PDEs with the hash pattern showing the proportion of inpatient readmissions for each diagnosis.

The 2 most common presentations were fluid overload and arrhythmia, with half of the fluid overload encounters resulting in readmission and a third of arrhythmias readmitted. We can see some trends in the indication for PDE between groups, but the only difference to reach significance was a greater percentage of open surgical patients who presented with arrhythmia. With cardiac NP monitoring, patients can often be guided to the hospital if there is a need for escalated care. This will frequently involve arranging transportation; $60 \%$ of all PDEs were NP guided. If the encounter was guided, the patient was more likely to present to NYU rather than their local hospital; $74 \%$ versus $33 \%$ of nonguided encounters. As a final point, we examined the effect of our RRIs on readmission rate for BPCI patients. In this study, there are 175 bundled participants. Compared with 2014 claims data, there is a significantly reduced rate of readmission from $18 \%$ to $11 \%$ after initiation of our RRI. There are, of course, several limitations to this study. It is a single-center experience, and our practice changes may not be feasible at other institutions. We had only 6 months of data available for analysis, so the sample size is relatively small. Although 90-day data are in the pipeline, this study followed patients for only 30 days. Claims data, as is the case with any administrative database, can be unreliable. It was difficult to corroborate details when patients were seen at other hospitals.

In conclusion, our approach to reengineering postdischarge management of BPCI valve patients under tight cardiac service line and cardiac NP control has significantly reduced costly 30-day readmissions in this high-risk population. The full fiscal impact of this strategy on BPCI valve patients will be understood next year when the 90-day claims data are reconciled. Additionally, further data and analysis may allow focusing postdischarge management resources on those patients identified at high risk for 30- and 90-day readmissions. We need to take ownership of the postdischarge period to survive in the era of financial risk sharing and we cannot rely on administrative databases but must create our own databases and processes. To satisfy my mentor, Dr Grassi's unique sense of humor, I leave you with an analogy from Douglas Adams' Hitchhiker's Guide to the Galaxy. Much like the secret dictatorship of mice over men, policy makers have inexplicable influence over clinical care decisions that dictate how we practice. Thank you.

Dr Fann. The discussion will be opened by Dr Robin Cohen.

Dr Cohen. Michael, that was an excellent presentation. I really enjoyed reading your paper. I have to say, and I'm standing next to Gene Grossi, his outfit says Aloha. Yours, not so much.

Dr Koeckert. Oh yeah. I'm still young.

Dr Cohen. I enjoyed reading your paper because I think you did a nice job of framing the quality cost paradigm that we all face on a daily basis: How do you decrease hospital stay and reduce SNF and rehab use without increasing hospital readmissions? How do you work with new payer mandates without annihilating your own margins with increased internal resource utilization? What kind of changes in clinical care protocols have the greatest chance of actually decreasing hospital admissions and making you look better in this new administrative paradigm? I'm sure that everyone in the room has their own strategies for dealing with these issues and I'm sure we'll hear from some of them this morning. I think you start with the low-hanging fruit and your $70 \%$ discharge rate to rehab or other facilities was pretty high. You had some easy work to do there. Like you, we've stepped up our PA follow-up with phone calls once the patients are discharged. We've found that having patients see their primary care doctor on day 3 has really decreased hospital readmissions in the first week for us. To me, replacing hospital readmissions with 48-hour urgent care observation doesn't really solve the problem. Eventually, this strategy won't go unnoticed by your quality manager, your hospital CFO, and eventually CMS.

I have 3 questions. First of all, when my wife and daughters go shopping, they show me everything they bought and rave about how they got it on sale. Sometimes we even get rebates from the credit card company but I know that I'm paying a big bill at the end of the month. Have you actually estimated what you've spent on PAs, nursing, physician time, 48-hour hospital or urgent care stays? In other words, how much are you willing to spend in order to save the payers money? Secondly, I appreciate your effort to take control of these patients by trying to bring them back into your system when they have postoperative issues. However, this needs to be a team approach, especially when TAVR is involved. What have you done to bring in your cardiologists and your primary care doctors so that you are preventing readmissions as a heart team and not just a surgical group? Finally, how do you use your data to further decrease the likelihood of hospital admissions? For instance, if atrial fibrillation and volume overload account for half of your 
readmissions, are you doing anything new that is helping you prevent those things? We've all struggled with atrial fib, but it would seem to me that volume overload should be something that we ought to be able to prevent in the first week. Thanks very much.

Dr Koeckert. Thank you, Dr Cohen. In response to your first question, in terms of actual dollars, I don't ... We haven't done that analysis and I don't have that data but I do know that we've essentially doubled the number of office visits and we have the phone calls, which is a new thing as part of this initiative so we've essentially increased our office staff by 2 full-time-equivalent NPs. We also reappropriated one of the clinical NPs to be a dedicated discharge NP who makes the phone calls in the first week and sets up all the discharge planning. In terms of the actual dollars spent and physician time, it would be hard to quantify but I don't have that data. To your second question ... Wait, what was your second question? [inaudible 00:34:44] Oh yeah. Right, thank you. Sorry. You're absolutely right, it should be a team approach and it is. You know, still on some level it's fragmented with the division between surgery and cardiology, but we do talk to the cardiologists when they see them. We try to get them all set up with their cardiology appointments before discharge so they're seen on day 14. Then, we follow-up with the cardiologist and make sure everybody is on the same page.

In response to your third question, this is relatively new data that we're collecting and while we do know that arrhythmias and fluid management are problems postoperatively, in terms of our response to the data that we've collected so far, things that we've done include looking more closely at the tele health data that we're collecting and this is kind of a brand new thing for us to have all patients set up with tele health, specifically Cardiocom and you know, with the daily weight trends, we can look at it and hopefully manage some of the diuretics as an outpatient as opposed to having somebody come in with CHF. In terms of the arrhythmias, you know, the vast majority are atrial fibrillation, of course, and that's kind of a cost of doing business of open heart surgery on some level. We do use routine beta-blocker prophylaxis. Our practice is not to use amio that frequently, but the thing that, I think, we have done well is one, when this problem occurs, to direct them to NYU and using the observational stay, and I know how you feel about that, it kind of streamlines the process to get them in, tuned up, and get them out a little bit quicker than if they were actually admitted to the inpatient floor, especially at an outside hospital where they would be running redundant tests, etc.

Dr Fann. Richard?
Dr Shemin. Richard Shemin from UCLA. You know, I think this is a very important study because you're part of a demonstration project. I think all of us are involved in 30-day readmissions for coronary artery bypass graft and others, so you've just given us a slice of the pie for valves but obviously our entire infrastructure has been focused on inpatient care and now, as we go at risk for 30 to 90 days postoperative care, you know, at UCLA, we've had to have many FTE's added in order to cover all of the phone calls, monitoring of tele health that we're also doing on a daily basis, and then providing postoperative outpatient management because we also not only try to prevent emergency room visits and readmissions, but go ahead and if you'll look at our data as far as the number of interactions when we changed our retic dose, antiarrhythmic therapy, and things like that, it's very, very large. Also, we transmit pictures of wounds and things like that. I'm interested in how do you get your hospital to support the infrastructure that's necessary to go ahead and provide all of this outpatient care. Traditionally, we cost shifted to another part of the health care system.

Dr Koeckert. Thank you and that's a great question and a little above my pay grade on some level. I think there's kind of a push from the administration side to move to this bundled payment model so I know that there's been a lot of support from them but aside from that, I'm not really privy to the upper management decisions.

Dr Fann. Eugene, a brief comment?

Dr Grossi. Brief comment is that we are a little fragmented in that we have 2 groups. We're doing the 30-day and there's a 31- to 90-day different management group as we undertook this on. We're now bringing the 2 together. For us, this is only a small piece. There's basically over a year, half a million on the table that could be leaking out in terms of dollars for both the TAVRs and another half million for the open surgical patients, so everybody realizes this is significant money. The question is, we're beginning to ... We're getting rebate checks so the administrators are very happy but that doesn't mean we're not losing money on the Medicare patients, but I think the real value is going to come as we develop these data and go through the 90 days, we're going to understand, is somebody pickpocketing us, taking money, the CMS taking money out of our pockets to do chronic disease management on patients, not to provide 90-day follow-up care for a surgical procedure. I think only by looking at these data very carefully are we going to be able to demonstrate are they just shuffling the deck and taking from us to give to somebody else. That's my thought on it.

Dr Fann. Very good. Thank you. Dr Koeckert, thank you very much for a very [crosstalk].

Dr Koeckert. Thank you. 